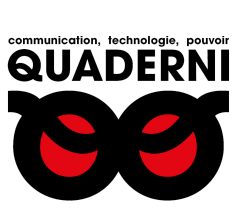

\title{
Quaderni
}

Communication, technologies, pouvoir

98 | Hiver 2018-2019

Humanités numériques : vers l'institutionnalisation

\section{Les humanités numériques, un enjeu historique}

Digital Humanities, an historical issue

\section{Frédéric Clavert et Valérie Schafer}

\section{(2) OpenEdition}

\section{Journals}

Édition électronique

URL : https://journals.openedition.org/quaderni/1417

DOI : 10.4000/quaderni.1417

ISSN : 2105-2956

\section{Éditeur}

Les éditions de la Maison des sciences de l'Homme

Édition imprimée

Date de publication : 5 février 2019

Pagination : 33-49

Référence électronique

Frédéric Clavert et Valérie Schafer, «Les humanités numériques, un enjeu historique », Quaderni [En ligne], 98 | Hiver 2018-2019, mis en ligne le 05 février 2021, consulté le 08 janvier 2022. URL : http:// journals.openedition.org/quaderni/1417 ; DOI : https://doi.org/10.4000/quaderni.1417 


\section{$D$ ossier}

\section{Les humanités numériques,} un enjeu
historique

Frédéric

Clavert

Valérie

Schafer

$\mathrm{C}^{2} \mathrm{DH}$

Université du Luxembourg
En 2011, à la réunion annuelle de l'American Historical Association, se tient une démonstration de Culturomics - un ensemble de méthodologies qui tente de saisir les tendances culturelles par l'analyse quantitative de très grands corpus textuels comme Google Books. Dans un billet intitulé « Loneliness and Freedom ${ }^{1}$ » le président de l'AHA, Anthony Grafton, tout en reconnaissant l'intérêt de cette démonstration, les perspectives immédiates, mais aussi les enjeux disciplinaires plus larges que ce vaste projet soulève, notamment pour la discipline historique, relève l'absence d'historiens, qui semblerait pourtant pertinente. Répondant à cette remarque, les deux directeurs du projet, Erez Aiden et Jean-Baptiste Michel, font un constat pessimiste de l'implication des historiens, notant le manque d'intérêt de certains, un regard négatif sur l'histoire quantitative des années 1950, un manque de compétences computationnelles ou encore des réticences face à un travail collaboratif multi-auteurs ${ }^{2}$.

Et pourtant, les historiens ne sont évidemment pas tous hostiles aux approches quantitatives et computationnelles et ont pu les intégrer dans leurs travaux depuis plusieurs décennies. En France, au sein de l'école des Annales, en cohérence avec la notion braudélienne de « longue durée ${ }^{3}$ ", de nombreuses recherches ont utilisé comme source de grandes séries statistiques en une approche quantitative reposant sur l'usage de l'informatique de l'époque. Si histoire économique et démographique quantitative sur données sérielles ont perdu en prestige dans les années 1970 - critiquées notamment par le mouvement de la micro-histoire - les approches et méthodologies quantitatives n'ont pas été aban- 
données, comme en témoignent la création de la revue Histoire \& Mesure dans les années 1980 et, plus récemment, la publication (et le succès) de Méthodes quantitatives pour l'historien ${ }^{4}$. Les grands débats autour des démarches quantitatives sont relancés aussi en interne, par exemple par l'History Manifesto ${ }^{5}$ et le débat qui s'en est suivi ${ }^{6}$. En outre les appels à la collaboration et à l'ouverture sont également renouvelés avec le développement des méthodes numériques, appelant à penser «une vision intégrée de la pratique historique à l'ère numérique qui souligne l'hybridité comme sa caractéristique principale $^{7} . »$

Les historiens ne sont pas en reste pour s'investir dans les humanités numériques (DH) et si d'autres disciplines à l'instar des sciences de l'information et de la communication (SIC) ont pu mener un effort collectif pour penser la relation à leur discipline (voir par exemple le « Manifeste pour un positionnement des Sciences de l'Information Communication (SIC) vis-à-vis des Digital Studies (DS) et autres mutations du Numérique $\left.{ }^{8} »\right)$, la réflexion historienne se saisit aussi de ces enjeux, notamment à travers des rencontres comme DH Nord dont le thème de 2017 portait sur « (Dé)construire l'histoire numérique ${ }^{9}$ " ou avec des ouvrages comme L'histoire contemporaine à l'ère numérique $^{10}$. Or, il est clair que non seulement le numérique et, dans le cas qui nous intéresse plus spécifiquement, les DH concernent les historiens à tous les stades de leur recherche, du rapport à l'archive et de la constitution des corpus à la diffusion de leurs travaux, mais ils modifient aussi les espaces de dialogue et de circulation des idées d'une partie de la communauté histo- rienne. En cela les DH sont bien un enjeu pour la fabrique et l'écriture de l'histoire, mais peuvent aussi à terme devenir un objet d'histoire. Si, dans cette relation entre $\mathrm{DH}$ et histoire, nous nous intéresserons plus modestement à ce que fait l'histoire aux $\mathrm{DH}$, bien qu'il y ait matière à analyser les échanges qui se nouent au sein de cette interdiscipline et le rôle qu'y tiennent ou n'y tiennent pas les historiens, c'est surtout sous l'angle des apports des DH à l'histoire que nous penserons une intégration et une hybridité à la fois (inter) et (intra) disciplinaire, au service non pas de la dilution disciplinaire mais de son enrichissement.

\section{Une ouverture (inter) et (intra) disciplinaire}

Ainsi que l'ont relevé aussi bien des membres convaincus des humanités numériques que certains de leurs critiques - car le développement des DH a fait débat, en venant bouleverser le champ institutionnel et académique - celles-ci sont une transdiscipline ${ }^{11}$, dont les périmètres sont sciemment larges et ouverts. Comme le notait J-C. Plantin, voisinant avec les humanities computing ${ }^{12}$, ou encore les computational social sciences ${ }^{13}$, "les digital humanities suscitent des définitions hétérogènes. Si une multiplicité des manières de se définir est commune à d'autres disciplines, et est un signe de réflexivité salutaire au sein d'une communauté de chercheur(e)s, le flou autour du nom digital humanities reflète un positionnement davantage stratégique. La flexibilité par rapport aux labels traduit en effet une volonté de vouloir laisser ouvert un champ des possibles sur ce que sont les digital humanities et ce que l'on peut $y$ faire $[\ldots]^{14}$. » Partant du livre de 
2005 de Willard McCarty, Aurélien Berra souligne cette ouverture, insistant derrière l'auteur de Humanities Computing sur cet « espace de dialogue, une zone d'échange dans laquelle se rencontrent les données et les méthodologies des différentes traditions savantes. Pour chaque praticien, elles sont aussi l'occasion d'une expérience réflexive instrumentée $[\ldots]^{15}$. » $\mathrm{Ce}$ pragmatisme n'est pas forcément opportuniste, mais relève aussi d'un refus d'établir une définition qui préexisterait à l'usage selon Pierre Mounier $^{16}$, qui s'est investi dès l'origine dans le développement francophone du mouvement. Les humanités numériques se pensent comme une zone d'échange ${ }^{17}$, un objet-frontière ${ }^{18}$, voire un projet-frontière, comme le propose Julia Bonnacorsi ${ }^{19}$ en partant d'une notion proposée par Morgan $\mathrm{Meyer}^{20}$. Le collectif et l'interdisciplinarité les caractérisent, mais d'autres valeurs relevées par Lisa Spiro ${ }^{21}$ offrent également matière à réflexion et éventuellement évolution des pratiques historiennes. Outre la collaboration, elle énumère en effet parmi les valeurs fondatrices des $\mathrm{DH}$, l'ouverture, la collégialité et la connectivité (auxquelles J.-C. Plantin ajoute le rôle des outils de communication pour relier les membres), ou encore la dimension expérimentale.

L'ouverture, entendue ici autant comme l'accès ouvert aux publications que le partage des outils et logiciels, réinterroge par exemple la diffusion des résultats, les espaces de publication et publicisation des travaux, le rapport à l'audience et au public des productions historiques, tandis que s'ouvrent des espaces de communication et de publication nouveaux. Les historiens voisinent désormais avec les sociologues, les cher- cheurs en SIC ou encore les linguistes dans des revues comme Digital Humanities Quaterly. Dans le numéro spécial francophone de cette dernière ${ }^{22}$, l'approche historienne de Crimino$\operatorname{corpus}^{23}$ ou l'article d'Anne Baillot ${ }^{24}$ côtoient musique, lettres et géographie. La réflexion sur l'accès ouvert, qui caractérise nombre des productions et publications des acteurs des $\mathrm{DH}, \mathrm{s}^{\prime}$ articule avec un mouvement plus large ${ }^{25}$, alors que les historiens sont incités, comme les autres disciplines, au dépôt de leurs productions dans des répertoires ouverts (type HAL), par ailleurs réclamés voire imposés par les institutions. Ils doivent aussi de plus en plus penser le partage ou au moins la « citabilité » de leurs sources, ce qui dans le cas du numérique peut poser plusieurs défis dont certains ont trouvé des solutions (développement de permaliens par la BnF pour pouvoir citer les archives du Web ou encore des DOI et autres identifiants pérennes, dans le champ des publications ${ }^{26}$ ).

Collégialité et connectivité ensuite, sans recouper totalement la notion d'ouverture, permettent aussi d'insister sur ces nouveaux espaces d'échanges, accueillants pour les historiens, que sont notamment des associations type Humanistica $^{27}$, ou encore les conférences DH, qui scandent l'année universitaire en Europe. Côtoyant de multiples disciplines, sujets et objets, dont témoigne par exemple le programme DH Benelux 201828, les historiens peuvent trouver là de nombreux points de contacts avec leurs collègues : la variété des approches en $\mathrm{DH}$, comme le montre le panorama qu'en dresse Alexandre Gefen en 201729, peut répondre à des interrogations en termes à la fois de recueil et gestion de sources et de données, d'analyse et 
de représentations via des outils numériques comme l'usage ou la constitution de bases de données, de cartographie de liens, d'étude sémantique ou lexicale, etc. - ou encore de valorisation, notamment dans le cadre de l'histoire numérique publique.

Enfin, les expérimentations, autre valeur que relève Lisa Spiro, font des DH un lieu de bricolage. Cette approche parfois artisanale peut inciter la discipline historique à une ouverture de ses coulisses, ainsi qu'à un regard réflexif renouvelé sur ses productions, éléments qui existaient auparavant, mais étaient rarement dévoilés au public. L'ouvrage Expérimenter les humanités numériques ${ }^{30}$ témoigne ainsi d'une volonté de penser la place du tâtonnement, de l'artisanat, des impasses dans les processus de recherches faisant écho au « manifeste pour les humanités numériques $2.0 »$, qui notait : « C'est le processus qui est le nouveau dieu vivant pas le produit. [...] Les humanités numériques se nourrissent de recherches itératives, des mobilisations collaboratives et de travail en réseaux. Elles valorisent la qualité des résultats mais aussi les étapes par lesquelles ceux-ci sont obtenus, qui représentent une forme de publication de valeur comparable. Le royaume $d u$ processus recèle des mines d'or inexploitées de savoir $^{31}$.»

Historicisant ce constat, Aurélien Berra le reformule en termes de prototypes et de démonstrations, invitant le chercheur à retrouver le goût historique de l'expérimentation : "Alors que nos institutions sont souvent peu propices à un renouvellement rapide des savoirs et des pratiques, nous vivons encore l'âge des prototypes.
Un historien a d'ailleurs suggéré de faire droit au temps des démonstrations : les sociétés savantes du XVIII siècle n'ont-elles pas posé les fondements des sciences modernes en faisant se côtoyer le sérieux et le douteux, le spectacle technique et l'expérience scientifique, sous le signe de l'expérimentation ${ }^{32}$ ?»

Si bien évidemment la production de résultats intermédiaires peut aussi être parfois le signe de conflits de temporalités entre le temps de la recherche et celui des financeurs et des projets, qui impliquent des livrables et publications réguliers, voire une communication sur la science en train de se faire pour des raisons d'échéanciers, le souci d'éclairer désormais les étapes de la recherche, peut aussi être de nature à intriquer plus étroitement l'histoire et son épistémologie.

En outre le dialogue interdisciplinaire stimulé par les DH a aussi des effets directs sur les relations au sein de la communauté historienne. Les séminaires organisés par le PIREH à Paris 1 autour de Stéphane Lamassé et Gaëtan Bonnot ${ }^{33}$ comme la conférence dédiée à l'histoire numérique organisée à Lille en 2017 deviennent des temps d'échange qui dépassent les thématiques et les périodes historiques, pour mettre en dialogue antiquisants, médiévistes, modernistes et contemporanéistes. Autour d'outils mais aussi de réflexions sur la notion de corpus, d'archives, ou encore les effets de leur numérisation, ils facilitent des échanges que la spécialisation sur un objet de recherche finit par faire perdre au fil des carrières. 


\section{Des enjeux transversaux à l'écriture de l'histoire}

Si les historiens peuvent dans le cadre des humanités numériques engager un dialogue non seulement intra mais aussi interdisciplinaire, c'est que les points d'échanges se jouent à plusieurs niveaux.

Le premier s'organise autour des outils, car les historiens utilisent comme certaines recherches en linguistique, en géographie ou en SIC par exemple, Gephi ou Iramuteq pour une partie de leur analyse. Iramuteq, logiciel libre implémentant la méthode Reiner ${ }^{34}$, permet d'analyser aussi bien des tweets dédiés aux commémorations de la Grande Guerre ${ }^{35}$ qu'un corpus sur les violences faites aux femmes pendant le génocide arménien ${ }^{36}$.

Un second niveau est constitué de notions comme celles de corpus, de données, d'archives qui sont notamment réinterrogées au prisme de la numérisation, de leur inflation ${ }^{37}$ ou de travaux sur le patrimoine nativement numérique ${ }^{38}$.

Enfin, un troisième niveau est structuré autour des médiations de la recherche, voire de ses médiatisations, deux enjeux qui, sans se recouper, posent des questions communes de mise en partage, d'éditorialisation, et devraient encore davantage être pensées qu'elles ne le sont aujourd'hui en termes de digital litteracy et de pédagogie à mettre en œuvre pour former au sein des parcours universitaires à ces enjeux.

Ces grands traits connaissent quelques nuances nationales au sein d'un mouvement internatio- nal, ce qu'esquissait Alexandre Gefen soulignant la nature davantage expérimentale des DH aux États-Unis, face à une approche plus théorique et tournée vers des questions de corpus, patrimoniales en France ${ }^{39}$.

Parmi les éléments qui peuvent engager l'ensemble de la communauté historienne, puisque cela touche au cœur de sa discipline, il y a la question des corpus et de la numérisation des archives. On peut lui lier sans l'y fondre celle du patrimoine nativement numérique, reconnu en un même mouvement que le patrimoine numérisé en 2003 par 1'Unesco ${ }^{40}$. Si les historiens n'ont bien sûr pas attendu les DH pour penser le document ou le corpus numérisé $e^{41}$, ils ne peuvent que gagner à croiser leur approche avec celle de disciplines connexes, ce dont témoignent par exemple les récents ouvrages de M. Treleani et B. Bachimont ${ }^{42}$, ou encore l'appel «Sémiotiques de l'archive » pour un numéro de la revue Signata ${ }^{43}$. Un mouvement ample s'est aussi développé notamment autour des archives du Web, et l'apport des historiens aux DH est ici clairement palpable, tant la communauté historienne porte largement ces questionnements depuis les travaux pionniers du danois Niels Brügger $^{44}$. Les historiens ont pu largement contribuer au dialogue interdisciplinaire, mais aussi introduire dans la réflexion historienne des apports des STS, des digital studies et media studies, ou des SIC, par exemple sur la notion d'éditorialisation ${ }^{45}$, de gouvernance ou d'objet frontière ${ }^{46}$, ou encore de médiatisation. Surtout quand la Wayback Machine d'Internet Archive propose d'explorer plus de 284 milliards de pages Web sauvegardées au fil du temps, l'historien se trouve confronté à des enjeux qui invitent 
à penser en quoi les propositions des humanités numériques peuvent aider sa recherche.

Or, les archives du Web plaident aussi pour la notion de trading zone dont Pierre Mounier après Svenson soulignait l'importance dans les humanités numériques : "Un des éléments les plus intéressants des humanités numériques est sans doute d'être un des rares lieux de circulation et d'échanges à l'intérieur d'un champ constamment menacé d'occlusion par l'hyperspécialisation et le marquage de territoire $^{47}$. » La zone d'échange peut ici être double, au sein des équipes de recherche, mais aussi entre archivistes et chercheurs : c'était le cas par exemple dans le projet ASAP (Archives Sauvegarde Attentats Paris) qui intégrait les membres de l'archivage dans la recherche $e^{48}$. De même c'est au contact des bibliothécaires et archivistes que le projet britannique BUDDAH pensait l'accueil d'une dizaine de jeunes chercheurs pour mener des travaux fondés sur les archives du $\mathrm{Web}^{49}$.

La proximité qui se noue entre archivistes, bibliothécaires et chercheurs à la faveur de projets est parmi les points notables, qui " témoignent tous du fait que les DH sont une opportunité à la fois pour les chercheurs et pour les bibliothécaires. De nombreux savoir-faire repris par les DH sont déjà présents dans le monde des bibliothèques, que cela soit la production des savoirs (les techniques de recherche et les ressources numériques) ou la diffusion de ces derniers (le rôle moteur joué par les bibliothèques dans les archives ouvertes et la numérisation des sources) $)^{50}$. " À la grande variété des échelles et méthodologies convoquées pour archiver le Web répond par ailleurs celle des méthodes pour l'analyser. Or la réflexion porte ici aussi sur les outils, qui rajoutent encore une couche de médiation à l'analyse et donnent matière à réflexions méthodologiques, par exemple dans les travaux liés à la reconstruction de Geocities $^{51}$ ou de domaines nationaux ${ }^{52}$. Cette production est parfois adossée aussi à de nouveaux outils de recherche. Ainsi certains travaux d'Anat Ben-David et al. sur la reconstitution du Web yougoslave passent par l'usage d'Anaconda ${ }^{53}$, mais il n'est pas besoin de faire l'histoire du numérique pour utiliser les outils numériques : on peut penser à l'emblématique projet porté par l'EPFL Venice Time Machine ${ }^{54}$.

Dans le même temps, c'est aussi la pratique de recherche concrète de l'historien qui est modifiée, comme l'a notamment mis en valeur Sean Takkats $^{55}$ qui « signale un premier changement évident : le mouvement du fauteuil vers les archives qui était autrefois de mise n'est plus puisque les archives sont consultables de chez soi. Et s'il peut y avoir un déplacement aux archives, lorsque celles-ci ne sont pas encore numérisées, alors le chercheur n'est plus assis à lire les documents, mais debout à prendre des dizaines de photos. Ce changement de paradigme a modifié notre façon de lire ", mais aussi notre rapport à la ressource, et notre imaginaire de la bibliothèque, comme le relève Xavier-Laurent Salvador ${ }^{56}$. Sean Takkats fait de la photographie numérique un symptôme «[...] puisqu'il s'agit bien souvent d'une collecte sans lecture ou d'une collecte suivie d'une lecture partielle. L'abondance de clichés pris par les chercheurs, qui craignent de devoir revenir aux archives ou de passer à côté d'une information importante 
nuit à leur analyse. Que fait-on avec toutes ces données? Comment les exploiter? Les rendre visibles? Les partager $?^{57} »$

Les historiens, qui se posent la question du goût de l'archive à l'ère numérique ${ }^{58}$, interrogent en une réflexivité accrue et une exploration des boîtes noires techniques, la raison computationnelle, le rôle des API, la déferlante d'images numérisées ou encore le rapport aux archives radiophoniques sous l'effet du numérique.

Tandis que "le processus de numérisation modifie les statuts et les valeurs culturelles des archives, non seulement du point de vue de l'accès aux collections mais également $d u$ point de vue des qualités matérielles et médiatiques des sources elles-mêmes (et par là, de la connaissance qu'elles rendent possible, scientifique comme profane) ", comme le note Julia Bonnacorsi $^{59}$, l'enjeu de la médiatisation dépasse celui des archives, alors que ce sont aussi de nouvelles formes de médiatisations des résultats de la recherche qui se font jour. Ces nouvelles formes de productions en réseaux peuvent accroître le nombre de contributeurs, élargir les publics, mais aussi inviter à une collaboration entre professionnels et amateurs, comme le souhaitent un certain nombre de projets de Digital Public History à l'instar du Guantánamo Memory Project ${ }^{60}$, ou de NC HB2: A Citizens' History ${ }^{61}$.

\section{À la recherche d'historicité}

Si les frictions entre temporalités du financement, de la recherche, de sa diffusion ont pu être soulignées plus haut, interroger les rapports aux temporalités des DH invite aussi à penser leur propre rapport à l'histoire : "Tout débat sur la cohérence épistémologique des humanités numériques s'appuie sur une narration, celle, plus ou moins précise, de la rencontre des sciences de l'interprétation avec cette nouvelle science qu'est l'informatique ${ }^{62} . »$

Et pourtant la recherche menée par Julianne Nyhan, Anne Welsh et Andrew Finn et entamée en 2013 autour d'une histoire orale des humanités numériques part du constat que, finalement, l'histoire des DH est peu connue ${ }^{63}$. Par les méthodes de l'histoire et, plus particulièrement, de l'histoire orale, ses porteurs espéraient pouvoir mettre en valeur les " histoires cachées » des humanités numériques. Publiés en $2016^{64}$, leurs résultats insistent en premier lieu sur l'idée qu'il faut regarder au-delà de l'université (et notamment vers l'industrie informatique) pour comprendre cette histoire. Dans les premières décennies de ce qui est d'abord des humanities computing, l'isolement des chercheurs revient régulièrement dans les interviews, ainsi que l'impression d'être révolutionnaires. Très centrés autour du monde anglo-saxon à peu d'exceptions près - dont l'historien Manfred Thaller - les auteurs de ce projet ne semblent pas noter l'émergence conjointe du quantitatif dans de nombreuses disciplines dans les années 1950 et 1960, de l'économie à la linguistique, en passant par l'histoire, notamment au sein de l'école des Annales ou de la cliométrie (la new economic history américaine).

Reste, comme le note Alexandre Gefen, des « ancêtres mythiques (le père Busa, jésuite pionnier de la numérisation et de l'analyse de corpus 
anciens), des gourous (le génial Franco Moretti, l'auteur de Graphes, cartes et arbres. Modèles abstraits pour une autre histoire de la littérature en 2008) $)^{65}$ ", qui sont largement convoqués. En effet, remarque Aurélien Berra ${ }^{66}$, de nombreux billets de blogs et des entrées Wikipédia ${ }^{67}$ font remonter les origines des humanités numériques à Roberto Busa, père jésuite désirant indexer les œuvres de saint Thomas d'Aquin grâce aux nouvelles capacités de ce qui, en France, est encore appelé « mécanographie ». Si cette histoire des origines est largement acceptée $e^{68}$, il ne faudrait pour autant ni la surestimer en une forme d'" héroïsation ${ }^{69}$ ", ni la sous-estimer. Au décès de Busa, Stephen Ramsay en évoquait l'influence sur son propre travail aux débuts de sa carrière ${ }^{70}$. Toutefois, comme le rappelle Ted Underwood, les « fondateurs » des humanités numériques semblent être plus nombreux et parfois moins connus ${ }^{71}$ et même, dans le cas de la lecture distante qu'Underwood analyse, leurs origines ne sont pas toujours liées à l'informatique.

Après Roberto Busa, la seconde grande pierre habituellement citée de l'histoire des humanités numériques est la Text Encoding Initiative (TEI). Née en 1987, dans un contexte de discussion autour de l'hypertexte, de l'internet et de la numérisation des sources, la TEI est en quelque sorte un concentré et une préfiguration des $\mathrm{DH}$ : par son souci de mettre les données (leur création, leur échange, leur pérennisation) au cœur de sa démarche, par son aspect « communautaire » (avec la création d'un consortium pour la faire évoluer en 2000) et, aussi, sa capacité à évoluer au fil des évolutions informatiques, y compris le web.
Enfin, Franco Moretti, devenu en une dizaine d'années peut-être aussi important que Roberto Busa, reste une autre référence incontournable avec son concept de lecture distante. Si l'on peut rappeler comme le fait Underwood que la lecture distante relève d'une généalogie relativement ancienne et pour partie non ancrée dans les humanités numériques, l'on peut également avancer que Moretti n'était pas le seul à vouloir théoriser un nouveau mode de lecture, fondé sur de grandes masses de données : dès le début des années 2000, par exemple, Stefan Sinclair et Geoffrey Rockwell, notamment autour du portail TaPOR mènent des travaux aboutissant, à partir de 2008, à Voyant Tools.

Dans cette quête des origines, commencent à se détacher certaines disciplines dont, par exemple, l'histoire numérique. Dans l'ouvrage Debates in the digital humanities coordonné par Lauren F. Klein et Matthew K. Gold, Stephen Robertson rappelle que les humanités numériques n'ont pas effacées les différences entre disciplines ${ }^{72}$ et l'historicité de chaque discipline au sein des humanités numériques peut être différente. Par ailleurs, le chapitre de Robertson refuse d'assimiler les DH aux études littéraires, les humanités numériques ayant été souvent rattachées aux départements d'Anglais dans les Universités américaines.

Si l'on regarde plus en détail le contenu du livre fondateur de Franco Moretti, Graphs, maps and trees, le terme « Graphs » fait par ailleurs référence à l'école des Annales. En se positionnant dans le cadre de l'École des Annales, Moretti n'agit pas au hasard. Dès 1959, François Furet et Adeline Daumard ${ }^{73}$ soulèvent le problème du 
big data de leur époque et répondent aux enjeux que posent de nouvelles sources en termes de masse - dans leur cas, les sources notariales - par l'informatique. Deux ans plus tard, les archéologues Paul Garelli et Jean-Claude Gardin s'interrogent sur une méthodologie informatique pour croiser des sources de données et, par ce croisement, obtenir de nouvelles informations ${ }^{74}$. Gérer des données massives et croiser des sources de données différentes : finalement, les fondements du big data sont posés depuis longtemps.

Une autre spécificité de l'histoire numérique est l'interrogation autour de l'historien programmeur, issue d'un célèbre article d'Emmanuel Le Roy Ladurie, publié en 1968 dans Le Nouvel Observateur $^{75}$. Le débat a fait l'objet d'un petit renouveau autour de 2011 et $2012^{76}$, mais n'a pas à être repris ici. Toutefois, l'un des cœurs, aujourd'hui, de l'histoire numérique est le site The Programming Historian - référence probable à Le Roy Ladurie. Développé depuis 2007 sous forme d'une introduction au langage de développement python pour les historiens, il décline des leçons, aujourd'hui très nombreuses, qui donnent une idée de la boîte à outils numérique des historiens.

Dans sa recherche d'historicité, les humanités numériques devraient toutefois connaître des évolutions, revaloriser et retrouver des figures on peut notamment penser à Paul Otlet ${ }^{77}$ ou Ted Nelson, l'inventeur du terme "hypertexte ». Comme le suggérait Jérome Valluy les premières années des $\mathrm{DH}$ ont fait la part belle à une approche techno-méthodologique portant moins sur les objets et davantage sur les méthodes, avant deux césures : en 2010 une rupture quan- titative tandis que "les informaticiens perdent ici une part du contrôle qu'ils avaient puisque le numérique s'adresse aussi aux profanes ${ }^{78} \gg$; 2013-2014 à l'occasion d'une réécriture de l'article « Humanités Numériques » de Wikipédia francophone ${ }^{79}$ et du développement du concept d'études digitales dans un ouvrage coordonné par Bernard Stiegler ${ }^{80}$, avant un tournant critique amorcé en 2015. La courte histoire des DH commence déjà à connaître une périodisation.

Alors que les pionniers ont posé des jalons importants - on pense ici au Manifeste des Digital Humanities rédigé à l'occasion du THATCamp Paris $2010^{81}$, que les disciplines ont pu introduire une nouvelle génération de manifestes ${ }^{82}$ et que des manifestes de seconde génération sont apparus $^{83}$, les early adopters voient l'arrivée de newbies, qui interroge sur l'évolution des périmètres et frontières aux contours souvent volontairement flous ou du moins largement inclusifs des DH. D'autant qu'il n'y a pas une, mais des communautés $\mathrm{DH}$, qui distinguent notamment des approches anglo-saxonnes et européennes, mais montrent aussi des tendances différentes au sein des pays européens que rendent palpables des rencontres comme DH Benelux ${ }^{84}$.

Autant de tournants qui peuvent faire évoluer à la fois les références des $\mathrm{DH}$, mais aussi peuvent à terme devenir objet d'histoire, pour qui à la croisée de l'histoire des sciences et de l'histoire du numérique voudra se pencher sur le riche matériel que les humanités numériques produisent. Ainsi alors que les DH ont eu à cœur d'articuler lieux de rencontres physiques (au sein des associations, des conférences DH, ainsi que des ThatHATcamps, depuis la tenue du premier en 
2008 à 1'Université George Mason et depuis 2010 pour le cas français) et débats en ligne, elles participent d'une forme d'organisation réticulaire, à l'instar des réseaux numériques abondamment exploités : "les digital humanities ne se contentent pas d'utiliser Internet, mais adoptent carrément la forme réticulaire d'Internet, à travers les liens entre plusieurs communautés distribuées, ou encore par l'absence de centralités a priori. De plus, les digital humanities se sont dotées dès le début d'un solide réseau de blogs de recherche, d'infrastructure de données, de codes partagés ou d'associations $^{85}$. »

Les humanités numériques ont aujourd'hui une histoire suffisamment ancienne pour avoir produit leurs propres archives, des sites web des THATCamps aux archives de listes de diffusion. Dans le cas francophone, par exemple, un atelier de THATCamp Paris 2015 a tenté d'exploiter les traces en ligne de ses précédentes éditions ${ }^{86}$. Les archives de la liste de diffusion des Humanités numériques francophones $[\mathrm{DH}]^{87}$, aujourd'hui service de l'association francophone pour les Humanités numériques Humanistica, mais créée à l'origine pour la préparation du THATCamp Paris 2010, sont une autre source de compréhension et d'analyse de la constitution, de l'évolution et de l'émergence des principaux acteurs des humanités numériques. Ces sources primaires pourront révéler les grandes étapes ${ }^{88}$, les controverses - par exemple, récemment, autour d'un texte de Marcello Vitali-Rosati ${ }^{89}$, notamment sur les réseaux sociaux numériques, mais également l'émergence des grands acteurs du champ. Ces archives, riche patrimoine nativement numérique, devront faire l'objet de cet aller-retour entre lecture distante et lecture proche que les humanités numériques ont placé au cœur de leur démarche.

\section{Conclusion}

Des éléments qui précèdent ressort que le numérique est exploité dans les $\mathrm{DH}$ « comme instrument de recherche; le numérique comme outil de communication; le numérique comme objet de recherche. C'est de ce complexe là que les Humanités Numériques se saisissent $[\ldots]^{90} \gg$ et c'est ici qu'est certainement aussi pour l'historien la valeur ajoutée, par rapport aux seuls usages de l'informatique au service de la recherche.

Dans tous les cas le constat effectué par Anthony Grafton dans « Loneliness and Freedom " semble aujourd'hui toujours plus d'actualité : «La vision traditionnelle du travail historique reflète encore les idéaux de Wilhelm von Humboldt, le fondateur de l'université moderne: "solitude et liberté». Comme la figure centrale dans Melencolia I de Dürer, l'historien est condamné à un travail intellectuel solitaire et incessant [...]. Pourtant, cette vision contient un élément de mythe. Chaque travail académique achevé, même s'il porte le nom d'un seul auteur, est le produit de nombreuses personnes travaillant ensemble de différentes manières ${ }^{91}$. »

Ainsi, au contact des humanités numériques, les historiens sont amenés à revisiter plusieurs aspects de la fabrique d'une histoire qui aujourd'hui articule productions individuelles et collectives, sous l'effet certes du numérique, mais aussi d'une culture du projet, qui innerve 
toutes les disciplines et peut être en partie reliée aux appels à financement. Les collectifs deviennent par ailleurs nécessaires à différents stades d'un projet, du recueil et de la numérisation de sources par exemple à la constitution de bases de données ou à la réalisation de sites collaboratifs, et les compétences requises dépassent les habituels savoir-faire disciplinaires, ce qui pose une nouvelle fois l'ancienne question de la formation informatique et numérique en Histoire ${ }^{92}$.

Toutefois, les historiens peuvent également faire valoir des compétences qui leur sont propres, la première étant leur maîtrise de la temporalité. La recherche d'historicité des humanités numériques n'est pas, aujourd'hui, sans poser problème : au-delà de la mythologie des précurseurs (Busa) et de la vénération des gourous (Moretti), les humanités numériques ont permis l'émergence d'un vaste et riche patrimoine numérique, sources primaires de leur propre histoire que les historiens pourront très clairement exploiter, par l'hybridation de leurs outils maîtrisés de longue date (la lecture proche) avec ceux des digital humanities, au premier rang desquels la lecture distante jouera un grand rôle.

Pour cette raison, l'historien ne doit pas craindre ce risque de «déterritorialisation » que soulevait Jean-Christophe Plantin. S'investir dans cette hybridation, pour reprendre les mots de Gerben Zaagsma, au sein d'une interdisciplinarité qui ne semble pas diluer les disciplines, leur permettra de continuer à tenir leur place au sein des enjeux scientifiques mais aussi sociétaux actuels. En cela nous suivons Xavier-Laurent Salvadorquinotait: «Lenumérique, aujourd'hui, constitue donc à n'en pas douter une seconde chance pour nos sociétés de remettre l'humain au centre du système et d'offrir un regard cultivé, diachronique $[\ldots]^{93} . »$ L'historien y a dès lors toute sa place, en un « tournant historique » ou une continuité que nous laissons aux historiens de demain le soin de qualifier. 
$\mathrm{N} \cdot \mathrm{O} \cdot \mathrm{T} \cdot \mathrm{E} \cdot \mathrm{S}$

1. A. Grafton, « Loneliness and Freedom », Perspectives on history, 03. 2011. https://www.historians. org/publications-and-directories/perspectives-on-history/march-2011/loneliness-and-freedom, consulté le 09.05.2018. Toutes les URLs citées dans ce texte ont été vérifiés le 9 mai 2018.

2. E. L. Aiden, J.-B. Michel, "Thoughts/Clarifications on Grafton's "Loneliness and Freedom" », Culturomics, 2011. http://www.culturomics.org/ Resources/faq/thoughts-clarifications-on-grafton-sloneliness-and-freedom

3. F. Braudel, " Histoire et Sciences sociales: La longue durée », in Annales. Économies, Sociétés, Civilisations 13 (4), 1958, p. 725-753.

https://doi.org/10.3406/ahess.1958.2781

4. C. Lemercier, C. Zalc, Méthodes quantitatives pour l'historien, Paris, La Découverte, 2008.

5. J. Guldi, D. Armitage, The History Manifesto, Cambridge, Cambridge University Press, 2014.

6. C. Lemercier, "Une histoire sans sciences sociales? ", in Annales. Histoire, Sciences sociales 2 (2), 2015, p. 345-357. http://www.revues. armand-colin.com/histoire/annales-histoire-sciencessociales/annales-histoire-sciences-sociales-ndeg22015-vol-70/histoire-sciences-sociales

7. "An integrative view of historical practice in the digital age that underscores hybridity as its main characteristic" in G. Zaagsma, « On Digital History », in BMGN - Low Countries Historical Review 128, 2013, p. 329.

8. F. Paquienséguy, « Manifeste pour un positionnement des Sciences de l'Information Communication (SIC) vis-à-vis des Digital Studies (DS) et autres mutations du Numérique ", in Revue française des sciences de l'information et de la communication
(10), 2017. http://journals.openedition.org/rfsic/2630 9. https://dhnord2017.sciencesconf.org

10. F. Clavert, S. Noiret, L'histoire contemporaine à l'ère numérique / Contemporary History in the Digital Age, Bruxelles, P.I.E.-Peter Lang S.A, 2013.

11.Voir notamment le carnet de recherche Humanlit d'Olivier Le Deuff et le "Manifeste des Digital humanities », ThatCamp Paris 2010, 2010, http://tcp. hypotheses.org/318

12. W. McCarty, Humanities computing, Basingstoke; New York, Palgrave Macmillan, 2005.

13. D. Lazer, A. Pentland, L. Adamic et al., « Life in the network: the coming age of computational social science », in Science (New York, N.Y.) 323 (5915), 2009, p. 721-723.

https://doi.org/10.1126/science.1167742

14. J.-C. Plantin, « Les Digital Humanities. Accomplissements et défis Pour un agencement postdisciplinaire ", in Les cahiers du numérique, 2014. http://lcn.revuesonline.com/article.jsp?articleId $=19808$

15. A. Berra, « Pour une histoire des humanités numériques », in Critique, $\mathrm{n}^{\circ}$ 819-820, 5/8, p. 613-626. 16. Cité in B. Caraco, « Les Digital humanities et les bibliothèques. Un partenariat naturel », in Bulletin des Bibliothèques de France (2), 2012. http://bbf.enssib.fr/consulter/bbf-2012-02-0069-002

17. P. Galison, "Trading Zone: Coordinating Action and Belief (1998 abridgment)", in M. Bagioli, The Science Studies Reader, Routledge, 1999, p 137-160. 18. S. L. Star, J. Griesemer, "Institutionnal ecology, 'Translations', and Boundary objects: amateurs and professionals on Berkeley's museum of vertrebate zoologie", in Social Studies of Science, 19(3), 1989, p. $387-420$.

19. J. Bonaccorsi, «Quelle réflexivité médiatique pour les Humanités Numériques ? La numérisation des manuscrits littéraires », in Les Enjeux de l'In- 
formation et de la Communication, $\mathrm{n}^{\circ} 16 / 2,2015$, p.83-98.http://lesenjeux.u-grenoble3.fr/2015-dossier/ 06-Bonaccorsi/

20. M. Meyer, «Objet-frontière ou Projet-frontière? », in Revue d'anthropologie des connaissances, 2009. http://www.cairn.info/revue-anthropologie-desconnaissances-2009-1-page-127.htm

21. L. Spiro, " "This Is Why We Fight": Defining the Values of the Digital Humanities », in M. K. Gold (éd.), Debates in the Digital Humanities, University of Minnesota Press, 2012, p. 1635. https://doi. org/10.5749/minnesota/9780816677948.003.0003

22. French Language Special Issue, Digital Humanities Quarterly, 2018. http://www.digitalhumanities. org/dhq/preview/index.html

23. M. Renneville, J.-L. Sanchez, S. Victorien, « Criminocorpus. Un projet numérique pour l'histoire de la justice », in Digital Humanities Quarterly 12 (1), 2018.

24. A. Baillot, « Reconstruire ce qui manque - ou le déconstruire? Approches numériques des sources historiques », ibid.

25. J. Farchy, P. Froissart, C. Méadel (dir.), « Sciences. com. Libre accès et science ouverte ", Hermès 57, 2010. https://www.cairn.info/revue-hermes-la-revue2010-2-p-9.htm

26. E. Bermès, « Des identifiants pérennes pour les ressources numériques: l'expérience de la $\mathrm{BnF} »$, International Preservation News (IFLA-PAC) (40), 2006, p. 1626.

27. http://www.humanisti.ca

28. http://2018.dhbenelux.org/draft-programme/detailed-programme/

29. A. Gefen, « Des humanités numériques en 2017 », Mélanges de la Casa de Velázquez, 47-2, 2017. http:// mcv.revues.org/7957

30. D. Martin, O. Legendre, E. Cavallié et al. (éds.), Expérimenter les Humanités numériques, Montréal,
Presses universitaires de Montréal, 2017.

31. Q. Julien, Y. Citton, « Manifeste pour des humanités numériques $2.0 »$, in Multitudes 59 (2), 2015, p. 181. https://doi.org/10.3917/mult.059.0181

32. A. Berra, op.cit.

33. Par exemple : Séminaire « Structuration et analyse de données historiques », https://www.pantheonsorbonne.fr/axe-de-recherche/pole-informatique-derecherche-et-denseignement-en-histoire/recherches/ seminaire-structuration-et-analyse-de-donnees-historiques/

34. M. Reinert, « Une méthode de classification descendante hiérarchique : application à l'analyse lexicale par contexte ", in Les cahiers de l'analyse des données 8 (2), 1983, p. 187-198.

35. F. Clavert, « Temporalités du Centenaire de la Grande Guerre sur Twitter », in V. Schafer (éd.), Temps et temporalités $d u$ web, Nanterre, Presses universitaires de Paris Nanterre, 2018, p. 113-134.

36. J. Chabot, M.-M. Doucet, S. Kasparian, « Témoigner malgré tout. Les récits des victimes du génocide des Arméniens face aux violences sexuées ", in Études arméniennes contemporaines (7), 2016. https://doi.org/10.4000/eac.987

37. Voir par exemple la réflexion engagée par la British Library autour des "Web Archives as Big Data » : http://blogs.bl.uk/webarchive/2015/09/tenyears-of-the-uk-web-archive-what-have-we-saved. html

38. À distinguer du patrimoine numérisé. Il s'agit notamment des archives du Web, de listes de discussions, de bases de données, etc., autant de matériaux existants « d'emblée » sous forme numérique.

39. S. Dumouchel, « Compte rendu de la séance introductive du séminaire EPHN », 19 mars 2018. https://movi.hypotheses.org/

40. Unesco, Charte sur la conservation du patrimoine numérique, 2003. http://portal.unesco.org/fr/ev.php- 
URL_ID $=17721 \& U R L \_D O=D O \_T O P I C \& U R L \_$ SECTION=201.html

41. Ainsi Jean-Philippe Genet proposait-il la notion de « métasource ». J.-P. Genet, « Histoire, Informatique, Mesure », in Histoire \& Mesure, volume 1, $\mathrm{n}^{\circ} 1$, 1986, p. 7-18. www.persee.fr/doc/hism_09821783_1986_num_1_1_904

42. M. Treleani, Qu'est-ce que le patrimoine numérique? Une sémiologie de la circulation des archives, Lormont, Le Bord de l'Eau, 2017 ; B. Bachimont, Patrimoine et numérique. Technique et politique de la mémoire, Bry-sur-Marne, Ina, collection Médias et humanités, 2017.

43. https://journals.openedition.org/signata/1447

44. Cf. notamment N. Brügger, « Digital Humanities in the 21st Century: Digital Material as a Driving Force ", in digital humanities quaterly, preview, vol. 10, n², 2016. http://www.digitalhumanities.org/dhq/ vol/10/3/000256/000256.html

45. Voir le support de présentation de L. Merzeau, « La médiatisation des archives : entre pratiques numériques et valorisation des fonds documentaires de l'audiovisuel, du Web et des médias ", séminaire udpn, 28 avril 2017. http://merzeau.net/la-mediatisation-des-archives-entre-pratiques-numeriques-et-valorisation-des-fonds-documentaires-de-laudiovisueldu-web-et-des-medias/

46. V. Schafer, F. Musiani, M. Borelli, "Negotiating the Web of the Past", in French Journal for Media Research, $\mathrm{n}^{\circ}$ 6, 2016. http://frenchjournalformediaresearch.com/lodel/index.php?id=952

47. P. Mounier, « Une 'utopie politique' pour les humanités numériques ?», in Socio - La nouvelle revue des sciences sociales, Paris, Éditions de la Maison des sciences de l'homme, 2015, p. 97-112. https://halshs. archives-ouvertes.fr/halshs-01142053/document

48. https://asap.hypotheses.org

49. J. Winters, "Breaking in to the mainstream: de- monstrating the value of Internet (and web) histories", in Internet Histories, 1-2, 2017, p. 173-179 ; J. Winters, "Coda: Web archives for humanities research - some reflections", in N. Brügger, R. Schroeder (eds), The Web as History, 2017, Londres, UCL Press, p. 238-248. Voir également : https://buddah. projects.history.ac.uk/

50. B. Caraco, op. cit.

51. I. Milligan, "Mining the 'Internet Graveyard': rethinking the historians' toolkit", in J. Can. Hist. Assoc. Revue de la Société historique du Canada 23(2), 2012, p. 21-64 ; I. Milligan, "Welcome to the web: The online community of GeoCities during the early years of the World Wide Web", in N. Brügger, R. Schroeder (eds), The Web as History, Londres, UCL Press, 2017, p. 137-157.

52. N. Brügger, D. Laursen, J. Nielsen, "Exploring the domain names of the Danish web", in N. Brügger, R. Schroeder (eds), op. cit., p. 238-248.

53. A. Ben-David, A. Amram, R. Bekkerman, "The colors of the national Web: visual data analysis of the historical Yugoslav Web domain", in International Journal on Digital Libraries, 2016.

54. https://vtm.epfl.ch

55. S. Dumouchel, «Compte rendu de la séance introduction du séminaire \#EPHN2017 (Invité : Sean Takats) », Billet, Modéliser et virtualiser, 18 janvier 2017. https://movi.hypotheses.org/129

56. X.-L. Salvador, « Contre l'éthique de l'algorithme : pour une éthique de la ressource », Le Monde Moderne, 20 avril 2018, https://www.lemondemoderne.media/contre-lethique-de-lalgorithme-pourune-ethique-de-la-ressource/

57. S. Dumouchel, op. cit.

58. Voir le livre en cours d'écriture, co-dirigé par C. Muller et F. Clavert : http://www.gout-numerique.net/ 59. J. Bonaccorsi, op. cit.

60. https://gitmomemory.org/ 
61. https://nchb2history.omeka.chass.ncsu.edu

62. M. Doueihi, «Quelles humanités numériques?», in Critique 2015/8, n 819-820, p. 704-711.

63. « About Hidden Histories: Digital Humanities 1949 - Present », http://hiddenhistories.omeka.net/ about

64. J. Nyhan, A. Flinn, Computation and the Humanities: Towards an Oral History of Digital Humanities, 1st edition, Springer, 2016.

65. A. Gefen, « Des humanités numériques en 2017 », in Mélanges de la Casa de Velázquez [En ligne], 47-2, 2017. http:// mov.revues.org/7957

66. A. Berra, op. cit.

67. À l'image de la Wikipédia francophone : « Humanités numériques », in Wikipédia, 17.04.2018. h t t p s : / / fr.wikipedia.org/w/index . php?title=Humanit\%C3\%A9s_num\%C3\%A9riques \&oldid $=147616937$.

68. Y compris chez l'un des auteurs : F. Clavert, O. Le Deuff, "Petite histoire des humanités digitales ", in O. Le Deuff (éd.), Le temps des humanités digitales, Limoges, FYP éditions, 2014.

69. J.-A. Bénel, «Quelle interdisciplinarité pour les " humanités numériques »? ", in Les Cahiers du numérique 2014/4, p. 103-132.

70. Le blog de Stephen Ramsay n'est plus accessible en ligne mais le billet est disponible sur la Wayback Machine : S. Ramsay, «Fr. Roberto Busa, S.J. (1913-2011) », 11.08.2011. https://web.archive. org/web/20121015012201/http://stephenramsay. us:80/2011/08/11/father-roberto-busa.html.

71. T. Underwood, «A Genealogy of Distant Reading », in Digital Humanities Quarterly 11 (2), 2017. 72. S. Robertson, «The Differences between Digital Humanities and Digital History », in M. K. Gold, L. F. Klein (eds.), Debates in the Digital Humanities 2016, Minnesota, Minnesota University Press, 2016. https://www.upress.umn.edu/book-division/books/ debates-in-the-digital-humanities-2016

73. F. Furet, A. Daumard, « Méthodes de l'Histoire sociale : les Archives notariales et la Mécanographie », in Annales ESC 14 (4), 1959, p. 676-693.

74. P. Garelli, J.-C. Gardin, « Étude par ordinateurs des établissements assyriens en Cappadoce ", in $\mathrm{An}$ nales ESC 16 (5), 1961, p. 837-876.

75. E. Le Roy Ladurie, " La fin des érudits », Le Nouvel Observateur, 08 mai 1968. http://hebdo.nouvelobs.com/sommaire/arts-spectacles/077609/la-findes-erudits.html

76. Voir par exemple THATCAMP PARIS 2012, " L'historien programmeur », in THATCamp Paris 2012 : non-actes de la non-conférence des humanités numériques, Paris, Éditions de la Maison des sciences de l'homme, 2012. http://books.openedition. org/editionsmsh/305 ainsi que F. Clavert, " Le code et l'historien contemporanéiste - pensées éparses ", Billet, in L'histoire contemporaine à l'ère numérique, https://histnum.hypotheses.org/385 et E. Ruiz, « Les historiens seront-ils finalement programmeurs ? », La boîte à outils des historien'ne:s, 22.09.2011, http:// www.boiteaoutils.info/2011/09/les-historiens-serontils-finalement/

77. Une journée d'études a d'ailleurs été consacrée à Paul Otlet et incluait une remise en perspective historique des Humanités numériques http://e3dlab.net/ prodigio/

78. S. Dumouchel, op. cit, 2018.

79. Cette refonte a été commencée lors d'un atelier de THATCamp Saint Malo. Voir J.-J. Hazoumé, O. Le Deuff, A. Serres et al., THATCamp Saint-Malo 2013 : Non actes de la non conférence, Paris, Éditions de la Maison des sciences de l'homme, 2014 (La Non-Collection). http://books.openedition.org/ editionsmsh/2181.

80. B. Stiegler (dir.), Digital studies : organologie des savoirs et technologies de la connaissance, Limoges, 
FYP Editions, 2014.

81. «Manifeste des Digital humanities », ThatCamp Paris 2010, 2010. http://tcp.hypotheses.org/318

82. F. Paquienséguy, op. cit.

83. Q. Julien, Y. Citton, op. cit.

84. http://www.dhbenelux.org/

85. J.-C. Plantin, op. cit.

86. «Les publications issues du DataSprint », Billet, THATCamp Paris. https://tcp.hypotheses.org/986

87. Voir https://groupes.renater.fr/sympa/arc/dh

88. Citons par exemple le message d'inauguration de la liste DH par Marin Dacos, le 23 mars 2010, « Bienvenue sur la liste Digital humanities - Humanités numériques ! », https://groupes.renater.fr/sympa/arc/ $\mathrm{dh} / 2010-03 / \mathrm{msg} 00000 . \mathrm{html}$

89. M. Vitali-Rosati, «Les chercheurs en SHS saventils écrire ? », The Conversation, 11 mars 2018. http:// theconversation.com/les-chercheurs-en-shs-saventils-ecrire-93024. On pourrait également citer le débat sur les fonctions de la liste DH elle-même, touchant également à certaines réalisations en infrastructures numériques, qui a eu lieu en octobre 2017 : J. Belmonti, « $[\mathrm{DH}]$ si tous les déabonnements du monde se donnaient la main... », 10 octobre 2017. https://groupes.renater.fr/sympa/arc/dh/2017-10/ msg00045.html

90. M. Dacos, P. Mounier, Humanités numériques. État des lieux et positionnement de la recherche française dans le contexte international, Paris, Institut français/ministère des Affaires étrangères pour l'action culturelle, 2014. http://www.institutfrancais. $\mathrm{com} /$ sites/default/files/if_humanites-numeriques.pdf 91. "The traditional vision of historical work still reflects the ideals of Wilhelm von Humboldt, the founder of the modern university: 'loneliness and freedom'. Like the central figure in Durer's Melencolia I, the historian is condemned to unremitting, solitary intellectual work, [...] time runs out. Yet this vision contains an element of myth. Every realized work of scholarship, even one that bears the name of a single author, is the product of many individuals working together in different ways" in A. Grafton, "Loneliness and Freedom », Perspectives on history, 2011. https://www.historians.org/publications-and-directories/perspectives-on-history/march-2011/lonelinessand-freedom

92. Question que le réseau e-NumHist, fondé en avril 2018, tente de relancer, en utilisant des méthodes chères aux Humanités numériques, à commencer par le carnet de recherche : https://enumhist.hypotheses. org/1. Dès 1993, Jean-Philippe Genet la qualifiait d'« urgente ». J.-P. Genet, « La formation informatique des historiens en France : une urgence ", Mémoire vive (9), 1993.

93. X.-L. Salvador, « Le numérique offre une seconde chance aux littéraires », slate.fr, 7 octobre 2016. http:// www.slate.fr/story/124818/numerique-litteraires 


\section{$R \cdot E ́ \cdot S \cdot U \cdot M \cdot E ́$}

Les historiens ne sont pas en reste pour s'investir dans les humanités numériques et si d'autres disciplines à l'instar des sciences de l'information et de la communication (SIC) ont pu mener un effort collectif pour penser la relation à leur discipline, la réflexion historienne se saisit aussi de ces enjeux. Il est clair que non seulement le numérique et, dans le cas qui nous intéresse plus spécifiquement, les DH concernent les historiens à tous les stades de leur recherche, du rapport à l'archive et de la constitution des corpus à la diffusion de leurs travaux, mais ils modifient aussi les espaces de dialogue et de circulation des idées d'une partie de la communauté historienne. En cela les DH sont bien un enjeu pour la fabrique et l'écriture de l'histoire, mais peuvent aussi à terme devenir un objet d'histoire. Si dans cette relation entre DH et histoire nous nous intéresserons plus modestement à ce que fait l'histoire aux $\mathrm{DH}$, bien qu'il y ait matière à analyser les échanges qui se nouent au sein de cette interdiscipline et le rôle qu'y tiennent ou n'y tiennent pas les historiens, c'est surtout sous l'angle des apports des DH à l'histoire que nous penserons une intégration et une hybridité à la fois (inter) et (intra) disciplinaire, au service non pas de la dilution disciplinaire mais de son enrichissement.

\begin{abstract}
Digital Humanities, an historical issue

Digital technologies may impact the practices of historians at every stages of their research, from retrieving archives, building a corpus to writing and editing. Digital tools have influenced spaces for scientific dialogue and free flow of ideas within the historians' community. As such, Digital Humanities are challenging the shaping of history, but they could
\end{abstract}

also become an object of history. Therefore, we will analyse such an hybridization that may enrich our field of study. 
